\title{
Evoluzione della politica scolastica sull'insuccesso e il disadattamento: il caso del Canton Ticino
}

\section{Edo Dozio}

L'articolo descrive l'evoluzione della politica scolastica sull'insuccesso e il disadattamento scolastico nel Canton Ticino durante gli ultimi 50 anni. In questo lasso di tempo vi è stata una importante evoluzione demografica e una profonda trasformazione economica. Il sistema scolastico adottato è stato di tipo integrativo e ciò ha profondamente influenzato soprattutto l'organizzazione della scuola secondaria inferiore. Dapprima la democratizzazione degli studi ha modificato il rapporto della società ticinese con la scuola; in seguito la revisione dell'insegnamento speciale e la creazione del Servizio di sostegno pedagogico hanno introdotto una nuova visione delle differenze nell'apprendimento e dell'insuccesso scolastico. Negli ultimi 30 anni, i Servizi creati si sono assestati e le concezioni e le pratiche attorno al disadattamento hanno subito ulteriori evoluzioni, ma all'orizzonte già si stanno profilando nuove esigenze di cambiamento.

La politica scolastica di un paese è la risultante di influenze diverse che subiscono nel tempo evoluzioni non sempre facilmente riconoscibili. Nell'articolo cercheremo di mettere in luce come la politica scolastica del Canton Ticino sia stata determinata negli anni ' 60 da elementi ideologici ed economici prima che pedagogici. Le strutture organizzate per far fronte all'insuccesso scolastico e al disadattamento si sono progressivamente stabilizzate all'interno di un sistema scolastico rinnovato. I cambiamenti sociali, le evoluzioni dei paradigmi esplicativi nelle scienze dell'educazione e la modifica delle richieste verso la scuola non sono riusciti che lentamente a trasformare le pratiche di insegnamento e di relazione all'interno della scuola malgrado questo sembri indispensabile affinché la scelta del principio politico dell'integrazione di tutti gli allievi in una unica scuola dell'obbligo possa essere realizzata. 


\section{L'inizio del modello integrativo (dagli anni '60 agli anni '70)}

L'epoca delle grandi riforme scolastiche che hanno coinvolto praticamente tutti gli ordini scolastici prende avvio nel Canton Ticino durante gli anni '60 e si realizza negli anni '70. Già nel 1957 il Messaggio del Consiglio di Stato al Gran Consiglio accompagnante il progetto di una nuova legge scolastica poneva il problema della democratizzazione degli studi nell'organizzazione scolastica postelementare. Il problema che ci si poneva allora non era formulato in termini di lotta o di riduzione dell'insuccesso scolastico, bensì di rendere possibile il reclutamento di intelligenze ovunque si trovassero. Le strutture scolastiche di quegli anni riducevano di molto le possibilità di formazione dei giovani ticinesi, a causa della separazione, dopo la scuola elementare, fra Scuola Maggiore e Ginnasio. Furono dunque adottate alcune misure organizzative affinché allievi socialmente o geograficamente meno favoriti potessero accedere agli studi. Iniziò così lo sviluppo del modello integrativo (Consiglio di Stato del Canton Ticino, 1957, p.5).

Gli anni '60 sono gli anni del boom demografico (dal '70 all' '80 la popolazione scolastica crescerà del 50\%) ed economico, dell'apertura del Ticino, del suo passaggio a un'economia sempre più terziarizzata. Inizia a farsi sentire la pressione affinché si disponga di persone formate e qualificate che possano contribuire allo sviluppo del cantone. La necessità di approfondire gli aspetti pedagogici relativi a questa nuova esigenza di formazione trova una formalizzazione istituzionale con la creazione in quegli anni di una Sezione Pedagogica dipartimentale. La riforma strutturale più rilevante fu l'istituzione della Scuola Media quale riforma della formazione postelementare: «occorre concepire tale periodo nell'ambito d'una formazione unica di base, rivolta allo sviluppo integrale del preadolescente, da attuare attraverso una scuola che offra veramente a tutti un'uguale base di partenza, cioè condizioni uguali di vita, di studio e di lavoro» (Consiglio di Stato del Canton Ticino, 1972, p. 6) I concetti che sottostavano a queste proposte erano il riconoscimento che le condizioni formative hanno un'influenza sullo sviluppo delle potenzialità degli allievi e che queste non devono essere dipendenti dall'origine sociale più o meno fortunata, così come il riconoscimento delle differenze individuali di sviluppo della persona e della sua intelligenza.

$\mathrm{Nel}$ Messaggio del Consiglio di Stato al Gran Consiglio concernente l'istituzione della Scuola Media nel Canton Ticino del 1972 si indicano alcuni problemi della Scuola Maggiore, quali l'eterogeneità delle classi: «(...) non potendo respingere o trascurare i ragazzi poco dotati d'intelligenza astratta, (la Scuola Maggiore) si è sempre trovata nell'obbligo di adattare i suoi programmi alle capacità dei singoli (inclusi gli allievi al di sotto della mediocrità, che nel ginnasio non sarebbero stati accolti o sarebbero stati bocciati più volte), in particolare dei giovani bisognosi di un'istruzione più pratica che teorica» (Consiglio di Stato del Canton Ticino, 1972, p.5-6). Il problema dell'insuccesso scolastico, o della selezione non 
democratica, come si sarebbe detto allora, preesisteva al momento della scelta fra Scuola Maggiore e Ginnasio. Il censimento scolastico del 1971 indica come il 25,6\% degli allievi che terminano la 5a elementare abbianno un ritardo scolastico di 1 o più anni (DPE, 1971). Oggi la percentuale è scesa al 6\% circa alla fine delle scuole elementari ed è del 15\% circa alla fine della scuola obbligatoria (DIC, 2000). Queste diminuzioni sono però controbilanciate dalla domanda di rinvio di un anno dell'inizio della scuola elementare, chiesta dai genitori ma anche dalle ispettrici della scuola dell'infanzia, che tocca circa il 10\% dei bambini. L'importanza della riuscita a scuola sembra portare oggi a un'anticipazione della selezione, contrariamente a quanto avviene nella Svizzera di lingua tedesca in cui si tende ad anticipare la scolarizzazione di qualche allievo a 6 anni invece dei 7 abituali.

L'obiettivo degli anni '60, promosso dapprima dalle associazioni magistrali e ripreso poi dall'istituzione, era di superare il concetto di scuola selettiva. Le ragioni e gli obiettivi della riforma del secondario erano nell'ordine:

- di tipo economico perché l'esplosione del settore terziario

[...] esige una formazione scolastica più approfondita che il secondario e questo è più esigente che il primario. [...] Ne consegue un impegno notevole della scuola per elevare il livello culturale e professionale di tutta la popolazione anche perché le competenze scolastiche richieste in ogni settore professionale aumentano (Consiglio di Stato del Canton Ticino, 1972, p. 32-33).

Gli obiettivi posti alla scuola dall'economia sono i seguenti:

a) Elevare il livello culturale e tecnico di tutta la popolazione. Anche il semplice operaio, al quale una volta si chiedeva una cultura generale limitata, dev'essere formato meglio nel periodo che precede la formazione professionale. Maggiori conoscenze matematiche, scientifiche e linguistiche sono necessarie per permettergli d'inoltrarsi con sicurezza in un mondo professionale sempre più tecnicizzato e mutevole. b) Favorire la formazione scolastica e professionale superiore. Ciò non significa rendere più facili gli studi. Bisogna invece eliminare tutti gli ostacoli che causano spreco di intelligenze [...] (Consiglio di Stato del Canton Ticino, 1972, p.36).

Quest'ultimo obiettivo è stato raggiunto considerando come oggi il tasso di allievi che ottengono, secondo i dati dell'Ufficio federale di statistica, la maturità liceale in Ticino (25.9\%) si situa al secondo posto in Svizzera dietro il Canton Ginevra (31.2\% nel 1998) con una media Svizzera del 17.5\% (nel 1932 i tassi erano del 3.3\% in Svizzera e del 2\% in Ticino, nel 1950 erano del 6.7\% in Svizzera e del 4.7\% in Ticino (Consiglio di Stato del Canton Ticino, 1972, p. 114)). - di tipo sociale come democratizzazione degli studi in quanto affrancamento della selezione scolastica dagli effetti dell'origine socio-economica, geografica e sessuale dei giovani. All'inizio del 2000, la discriminazione geografica e sessuale è stata praticamente annullata con l'istituzione di scuole medie in ogni valle del cantone e la moltiplicazione dei Licei. Per quanto concerne invece la differenza fra maschi e femmine, la riuscita delle ragazze alla Scuola Media è superiore a quella dei maschi (maggior percentuale di ragazze presenti nei 
curricoli elevati delle materie a livello). D'altra parte in Svizzera dal 1992 si è rovesciato il rapporto fra maschi e femmine nell'ottenimento della maturità. Rimane invece ancora presente l'effetto selettivo dell'origine socio-economica e culturale, anche se le popolazioni sfavorite non sono più le stesse di 30 anni fa vista l'ampiezza del fenomeno dell'immigrazione da regioni anche culturalmente distanti. Non esistono dati statistici recenti in merito; possiamo però segnalare come nella Scuola Media, la percentuale di allievi di cittadinanza svizzera nei curricoli più bassi è del $55 \%$ circa (su una presenza del $71 \%$ nella popolazione scolastica) mentre gli stranieri sono rappresentati nella misura del 45\% (29\% nella popolazione scolastica).

- di tipo psico-pedagogico in quanto la selezione scolastica avveniva troppo precocemente alla fine della scuola elementare e a troppi allievi con buone possibilità veniva impedito l'accesso agli studi superiori.

Le misure generali di miglioramento dell'istituzione scolastica previste per gli anni '70 erano:

evitare nella misura del possibile le ripetizioni di classi (se il 15\% degli allievi che frequentavano la scuola elementare aveva ripetuto 1 o più classi, la percentuale era del 27,2 per la scuola maggiore e del 4,3 per il ginnasio), recuperare gli allievi deboli in qualche materia, aiutare i giovani il cui ambiente familiare è povero di stimoli educativi e culturali, condurre anche gli allievi migliori ad attività che li impegnino a fondo (Consiglio di Stato del Canton Ticino, 1972, p. 10-25).

Era quindi necessario intervenire ben prima della separazione degli allievi nei due tipi di scuola alla fine delle elementari. La selezione sociale agiva già nelle età più precoci (Consiglio di Stato del Canton Ticino, 1972, p. 10-25).

Negli anni '70 solo il 56\% degli allievi di 5 anni frequentavano le Case dei bambini; dal 1990 praticamente il $100 \%$ dei bambini frequenta quella stessa istituzione, denominata però ora scuola dell'infanzia. Accanto al miglioramento della qualità dell'insegnamento, l'aumento del numero di bambini che frequentava l'asilo, la riduzione del numero dei bambini affidati ad una singola maestra (non più di 30) e l'individuazione precoce delle anomalie di sviluppo e delle carenze ambientali, fisiche, intellettuali e caratteriali erano ritenuti gli obiettivi da raggiungere per ridurre la selettività sociale che si sarebbe manifestata più avanti nella scolarità (allegato del DPE al Messaggio del Consiglio di Stato del Canton Ticino, 1972, p.91).

Per la scuola elementare non si prevedevano cambiamenti strutturali. Dal 1959 l'insegnamento era organizzato per cicli e i miglioramenti proposti negli anni '70 si concentravano sulla revisione dei programmi di insegnamento, la riduzione a non più di venticinque del numero di allievi per sezione, l'inizio dell'insegnamento di una seconda lingua e sui rinnovamenti metodologici e in particolare l'individualizzazione dell'insegnamento allo scopo, fra gli altri, di "consentire il ricupero degli allievi meno dotati» (Consiglio di Stato del Canton Ticino, 1972, p.92). 
Per quanto concerne l'insegnamento speciale, è nell'anno scolastico 1900/01 che a Lugano si apre la prima classe di questo tipo, ma per tutta la prima metà del secolo le iniziative di insegnamento specializzato sono lasciate ai comuni o a fondazioni private generalmente religiose. Alla fine degli anni '60, uno studio di C. Ferrari e W. Sargenti (1968) rileva come il Ticino avesse un tasso di scolarizzazione speciale molto basso secondo le definizioni dell'Assicurazione Invalidità: $1,6 \%$ in rapporto al 4,5 nel resto della Svizzera. Lo sviluppo dell'insegnamento speciale era previsto in due direzioni: creazione di scuole speciali destinate ad allievi con insufficienze mentali spiccate (quoziente intellettuale inferiore a 75) e creazione nelle sedi di scuola elementare di classi di osservazione e di introduzione destinate ad allievi immaturi o con difficoltà d'adattamento nei normali cicli di studio. Lo scopo di queste ultime classi sarebbe stato il reinserimento dell'allievo nell'insegnamento normale.

La riforma dell'insegnamento speciale degli anni '70 si fonda in larga parte sulla forza di giovani docenti formati in pedagogia curativa. Essi porteranno un contributo essenziale all'impostazione dei rinnovamenti e saranno promotori di progetti che troveranno sbocco dapprima in alcune sperimentazioni e poi nella Legge sulle Scuole Speciali. Erano le nuove metodologie della pedagogia attiva e le teorie psico-pedagogiche sull'origine delle difficoltà di apprendimento a entusiasmare questi docenti protesi a ridurre l'insuccesso degli allievi più sfortunati. La proposta di riforma della Scuola Media poneva con urgenza la necessità di riformare le Scuole Speciali «poiché gli allievi incapaci di seguire normalmente le classi dei vari cicli della scuola obbligatoria devono ricevere un insegnamento adatto alle loro capacità limitate, dal quale possano trarre il miglior profitto» (Messaggio, 1972, p.93).

Dalla descrizione precedente si può vedere come, in Ticino, alla base dell'evoluzione delle concezioni sull'insegnamento speciale, sull'integrazione invece che sull'esclusione -si ricordino le proposte di Basaglia (1973) in Italia- vi fossero innanzitutto la sensibilità e le intuizioni di tipo politico tipiche degli anni Sessanta. Solo progressivamente il discorso teorico prenderà spessore, grazie anche all'arrivo di nuovi giovani universitari formatisi in psicologia cognitiva, psicologia analitica, sociologia e pedagogia ed informati di pedagogia attiva, di pedagogia compensatoria, di descolarizzazione, ecc.

\section{Lo sviluppo del modello integrativo (la fine degli anni '70 e l'inizio degli anni '80)}

Con gli ideali integrativi nasce l'idea di un sostegno pedagogico inserito nelle istituzioni scolastiche, dalla scuola materna al secondario (Martinoni, 1990). Nell'anno scolastico 1979-'80 prende avvio l'attività dei Servizi di sostegno pedagogico nella scuola ticinese (SSP), sia nelle elementari, sia nella scuola media. Il primo scopo del Servizio di sostegno pedagogico era di fornire un aiuto agli al- 
lievi in grave difficoltà scolastica o di inserimento scolastico e, se possibile, di prevenire l'apparizione delle diverse forme di disadattamento. La scuola media in particolare stava muovendo i primi passi e doveva affrontare i problemi dell'integrazione, in un' unica struttura, di tutti gli allievi della fascia d'età fra gli 11 e i 15 anni. L'eterogeneità accresciuta della nuova scuola lasciava supporre che un certo numero di allievi avrebbe potuto incontrare delle difficoltà di adattamento o scolastiche; l'aiuto al superamento di queste difficoltà incombeva al Servizio in collaborazione con gli altri partner scolastici. In effetti, il Ticino era già allora un caso unico in Svizzera: solo l'1,5\% dei ragazzi in età di obbligo scolastico non frequentava una scolarità normale. Solo i cosiddetti casi $A I$, cioè gli allievi al beneficio di un'assicurazione di invalidità, erano inseriti nelle strutture di educazione speciale, contrariamente a tutti gli altri cantoni che ancora oggi hanno tassi di scolarizzazione in classi diverse da quelle ordinarie fra il 5 e il $20 \%$ ca. (SturnyBossart, 1996, 2000; Merzaghi, 1997).

Fra gli anni ' 40 e ' 60 , qualche comune ticinese già aveva istituito delle classi parallele nella scuola elementare. Con gli anni ci si era però accorti di come esse finissero per non soddisfare gli obiettivi per le quali erano state create; diventavano poco stimolanti per gli allievi, non favorivano il loro rientro nel curricolo normale ed erano a volte anche difficili da condurre. La separazione degli allievi dal gruppo della classe non favoriva il loro recupero bensì il loro adattamento a condizioni diverse e più protette. Alcune ricerche hanno approfondito questo tema confermando questi dati esperienziali. Se da una parte gli allievi che sono scolarizzati in situazioni protette vivono più tranquillamente la loro situazione e acquisiscono una buona fiducia di base, dall'altra parte il contrasto con il reale, all'uscita dalla classe protetta, risulta problematico poiché la mancanza di confronto non stimola quel recupero che si voleva favorire con la creazione delle classi parallele o di recupero (Bless 1990, Pierrehumbert 1992, Dozio 1993).

Negli anni successivi, alcuni docenti del settore primario furono incaricati di sperimentare delle forme di recupero individualizzato che sostituissero le precedenti classi di recupero. Gli allievi erano allora mantenuti nelle classi ordinarie e seguiti da un docente, individualmente $\mathrm{o}$ a piccoli gruppi, per una o più ore alla settimana.

Sarà nel 1984-' 85 che la nuova legge sui Servizi di sostegno della scuola materna, elementare e della scuola media verrà approvata ed i servizi progressivamente generalizzati a tutto il cantone. Nei documenti relativi alle sperimentazioni svolte e nel Messaggio concernente l'istituzione del Sostegno pedagogico troviamo l'essenziale delle riflessioni svolte nel corso degli anni '70:

le difficoltà di adattamento e di apprendimento scolastico non sono un fenomeno recente, ma hanno caratterizzato la scuola pubblica obbligatoria fin dalla sua costituzione: una parte degli allievi non riesce ad adattarsi alle norme di comportamento e ai programmi di apprendimento proposti dalla scuola (Consiglio di Stato del Canton Ticino, 1984, p.1). 
Le soluzioni fin qui ricercate dalla scuola si riferivano a tre modelli di intervento: ripetizione delle classi (in media il 19\% degli allievi era in quegli anni in ritardo scolastico), organizzazione di classi di recupero e organizzazione di interventi di aiuto individuale. La ripetizione si era rivelata non sempre utile, anzi spesso fonte di ulteriore disadattamento, e non rimuoveva i veri ostacoli all'apprendimento; le classi parallele si erano rivelate scarsamente stimolanti e favorivano l'adattamento dell'allievo a un nuovo standard costituito dal gruppo di allievi della classe parallela. Non restava allora che esplorare altre forme di sostegno fondate sui seguenti principi:

- mantenere l'allievo nel gruppo originale dei coetanei, quale espressione di accettazione dell'allievo stesso pur con le sue difficoltà;

- realizzare interventi individualizzati o a piccoli gruppi puntando sulle cause reali delle difficoltà;

- integrare gli interventi individualizzati con opportuni accorgimenti da effettuare in classe, durante le lezioni regolari, per evitare discrepanze e contraddizioni nell'azione educativa.

Non da ultimo è da segnalare come la soluzione sostegno pedagogico fosse considerata la più razionale ma anche la meno costosa per affrontare il disadattamento scolastico in modo efficace e valido! (Consiglio di Stato del Canton Ticino, 1984, p.2)

Nella Scuola materna e elementare il Servizio di sostegno pedagogico nasce con le figure di docente di sostegno, di logopedista e di psicomotricista, coordinati da un capogruppo che interviene anche nella scuola materna. Nel settore secondario assume innanzitutto il compito di far fronte all'eterogeneità della neocreata Scuola Media concepita secondo un modello integrativo di tutti gli allievi in età di scuola secondaria, tendente a posticipare il più possibile la selettività scolastica e mantenendo il più a lungo possibile gli allievi nel loro curricolo normale. "La realizzazione di una tale modifica implica evidentemente l'apparire di problemi nell'allievo fragile, ma anche nell'insegnante che non sempre è in grado di proporre risposte educative adeguate.»(Thurler, 1980, p.1). Anche nella Scuola elementare il sostegno pedagogico è introdotto per far fronte ai problemi di efficacia dell'insegnamento e prevenire il disadattamento, per permettere a tutti gli allievi, anche agli «allievi limitati nelle loro capacità di trarre profitto dagli scambi con i coetanei e dagli stimoli della scuola, pur senza pretendere di raggiungere sul piano dell'apprendimento scolastico tutti gli obiettivi minimi fissati dai programmi» (Consiglio di Stato del Canton Ticino, 1984, p.4).

Il Servizio viene costituito come servizio interno alla scuola e non come una struttura esterna alla quale la scuola deleghi l'attività con allievi in difficoltà. Se nei confronti dell'allievo ha lo scopo di permettere una frequenza regolare al maggior numero possibile di ragazzi, nei confronti dell'istituzione deve pure garantire un intervento tempestivo in modo da permettere alla scuola di assumersi compiutamente la responsabilità educativa di tutti i suoi allievi. Si cercherà quindi di creare all'interno della scuola stessa le condizioni di continuità dall'età 
prescolare fino all'inserimento professionale affinché tendenzialmente tutti gli allievi vengano sostenuti negli interventi educativi e rieducativi secondo premesse pedagogiche e di economicità rispetto alle altre soluzioni possibili.

In queste descrizioni si nota come al centro delle preoccupazioni vi fosse il desiderio di intervenire a migliorare l'allievo: il concetto di insuccesso scolastico era ancora prioritariamente riferito alle risorse dell'allievo stesso; sono i limiti dell'allievo a determinare il suo insuccesso e non vengono ancora messi in discussione i valori proposti dalla scuola o le sue metodologie. Si afferma il principio dell'adattamento dell'insegnamento alle caratteristiche degli allievi (carenze o disturbi individuali) proponendo l'individualizzazione dell'insegnamento come metodica affinché si aumenti la probabilità dell'apprendimento. La conferma di tale interpretazione la troviamo a pag. 1 del Rapporto della Commissione speciale scolastica del 1984: «L'avvenire scolastico è stato considerato come dipendente principalmente dalle loro caratteristiche individuali». Le ragioni della creazione di disadattamento scolastico sono successivamente riferite anche all'ambiente socio-culturale di origine degli allievi che la scuola dovrebbe tentare di compensare. La stessa Commissione scrive che l'interpretazione psicologica dell'attribuzione alle caratteristiche del singolo allievo delle cause dell'insuccesso ha rappresentato una tappa necessaria, e positiva, nel modo di considerare la vita scolastica. Ha reso possibile il venir meno di giudizi morali sulle difficoltà e sui fallimenti degli allievi: invece di imputare $i$ risultati negativi unicamente alla loro cattiva volontà o alla loro pigrizia si è tentato di trovare una spiegazione scientifica del loro comportamento e quindi di creare una serie di strumenti e di strategie che ne permettessero il superamento. In questa prospettiva il quesito posto è stato quello di trovare nelle caratteristiche individuali dell'alunno le cause delle sue difficoltà scolastiche (Commissione cantonale di coordinamento, 1987, p.1).

Negli anni ' 70 però tale ipotesi esplicativa viene superata: «non si è più vincolati ad un' interpretazione del disadattamento scolastico dovuto essenzialmente a motivi di ordine biologico, interpretazione secondo la quale l'insuccesso è insito nell'allievo stesso» (Commissione cantonale di coordinamento, 1987, p.1).

Dalla soluzione della bocciatura dell'allievo impossibilitato a soddisfare le esigenze della scuola, si passa ad un nuovo paradigma proposto e sostenuto dagli autori delle prime sperimentazioni di sostegno pedagogico:

dettagliate indagini metodologiche e pedagogiche hanno però da tempo confermato come esista pure una correlazione tra la buona riuscita scolastica e l'ambiente, familiare e non, che circonda l'allievo. Limitati stimoli intellettuali, povertà di linguaggio, culture notevolmente differenziate da quelle che la scuola propone, aumentano in modo tangibile il rischio di insuccesso (Commissione cantonale di coordinamento, 1987, p. 2).

Per quanto concerne la riflessione sull'istituzione scolastica, già nel 1979, E. Bennati-Besozzi scriveva che, con la creazione del Servizio di sostegno pedagogico, eliminando le classi di recupero, si riportano il ritardo e l'insuccesso scolastico all'interno della realtà scolastica in cui si sono evidenziati: il ritardo, il disadat- 
tamento viene considerato un problema della scuola e proprio per questo deve trovare una soluzione al suo interno. Se ciò non avviene, si deve parlare di carenza dell'istituzione scuola a rispondere adeguatamente ai bisogni individuali e sociali $e$ ad adattarsi alla realtà dei bambini che la frequentano (Bennati-Besozzi, 1979, p.5).

L'evoluzione delle riflessioni teoriche sul disadattamento scolastico, sulle sue cause e sui rimedi possibili, porta a una sensibilizzazione dei docenti al fenomeno e una condivisione sociale dei problemi legati all'insuccesso. Ne consegue una maggior tolleranza verso gli allievi con problemi di adattamento, una progressiva diminuzione del tasso di bocciature e di ripetizioni di classe. Gli operatori di sostegno pedagogico procedono a una esplicita categorizzazione dei problemi posti dagli allievi e alla ricerca di rimedi psicologici o pedagogici congruenti. A titolo di esempio, il Gruppo sperimentale del VI circondario di scuola elementare (1982) distingue: difficoltà socio-affettive importanti (52\% dei casi segnalati, di cui quasi la metà ha richiesto un intervento specialistico), difficoltà psicomotorie $(21 \%)$, problemi cognitivi $(10 \%)$, problemi organici $(9.5 \%)$, ritardo e difficoltà di linguaggio (28\%), difficoltà nel linguaggio scritto $(70 \%)$, difficoltà logico-matematiche $(54 \%)$ e allievi segnalati senza difficoltà di apprendimento scolastico (15\%) (Commissione speciale scolastica, 1984, p.3).

Durante gli anni ' 80 , i servizi di sostegno ormai costituiti e generalizzati lavorano essenzialmente secondo due principi: identificare nel modo più preciso possibile le cause delle difficoltà manifestate dagli allievi e intervenire, all'interno della scuola o facendo capo a servizi esterni, con terapie specifiche. Nel caso di carenze di stimoli sociali e ambientali si lavora nella logica della pedagogia compensatoria, promuovendo programmi di stimolazione cognitiva. Grazie alle statistiche sul funzionamento del servizio si possono conoscere alcune caratteristiche dell'insuccesso scolastico: i casi esaminati sono per il 60\% maschi (nella scuola media i maschi non promossi sono il 5,3\% mentre le femmine sono solo l'1,8\%), stranieri e svizzeri sono seguiti nella scuola materna in una proporzione molto vicina alla composizione della popolazione globale mentre nella scuola media gli stranieri segnalati sono il $40.7 \%$ su una percentuale del $27.4 \%$ nella popolazione globale, gli allievi appartenenti ai ceti socio-culturali inferiori sono sovra-rappresentati (64\% nella scuola media su una percentuale del $41 \%$ nella popolazione globale).

Le riflessioni sull'utilità di modificare l'impostazione dell'insegnamento per ridurre il disadattamento sono per il momento ancora scarse. Nel settore medio si propone di rendere universitaria la formazione dei docenti e di affiancare loro degli esperti disciplinari che garantiscano una migliore scientificità degli argomenti proposti; non vengono invece fatti riferimenti alle necessità di adattamenti della didattica. Il funzionamento del sistema come variabile che rivela forme di disadattamento, comincia a essere oggetto di riflessione con l'entrata in funzione dei primi gruppi di sostegno pedagogico. «L'approccio più corretto del problema del disadattamento scolastico pare dunque consistere nel considerarlo come un 
rapporto perturbato/conflittuale tra le diverse componenti che agiscono in modo interdipendente nella realtà scolastica [...]»(Thurler, 1980, p. 5). L'approccio che introduce in ambito pedagogico-didattico gli aspetti psicodiagnostici trova una formalizzazione esplicita. L'analisi delle situazioni segnalate avviene secondo tre dimensioni: localizzazione del disadattamento (genere di disadattamento, persone/oggetti implicati, strutture psichiche colpite), ricerca della causa del conflitto (cause intrinseche alla scuola o estrinseche - famiglia, ambiente sociale - e definizione di un progetto di intervento (diretto sui campi pedagogici ed extra-pedagogici del conflitto) (Thurler, 1980, p.7). Contatti e collaborazioni vengono istaurate, ogni volta che lo si ritiene necessario, con gli altri servizi di sostegno ed in modo sistematico con le scuole precedenti e seguenti. Queste modalità fondamentali di lavoro caratterizzeranno il servizio per tutto il decennio successivo. I docenti di sostegno svolgeranno diagnosi fondandosi su strumenti largamente ripresi dalla psicologia clinica e sociale e cercheranno delle collaborazioni di intervento con i docenti ed i consigli di classe.

La psicologizzazione delle analisi, l'uso di test e la ricerca di collaborazioni che mirano a introdurre delle modifiche nella didattica degli insegnanti si scontrano con le concezioni dei docenti sulle cause dell'insuccesso e del disadattamento scolastico. In larga maggioranza i docenti di materia concepivano e concepiscono tuttora l'insuccesso come mancanza di capacità o di buona volontà da parte dell'allievo e cercano di delegare all'operatore di sostegno la responsabilità di rinormalizzare l'allievo, proponendogli principalmente interventi di recupero scolastico che mirano a permettere al docente stesso di continuare il suo insegnamento senza dover troppo diversificare il lavoro in classe. Il rapporto Thurler (1980) conclude affermando come il lavoro di sostegno sia difficile proprio perché tocca aspetti molto diversi ed è molto dipendente dalla volontà di collaborazione di tutti gli agenti scolastici, della famiglia e dell'allievo stesso. Si ammette così che il disadattamento è un fenomeno complesso che spesso viene semplificato attribuendone le cause all'esterno della scuola (disturbi psicoaffettivi e capacità limitate dei bambini o carenze culturali causate dall'ambiente di provenienza), ma che raramente "si assume la possibilità che sia la scuola stessa a creare disadattamento e a essere incapace di rispondere ai bisogni eterogenei della popolazione scolastica» (Bennati-Besozzi, 1979, p.6). Si tratta quindi non solo di fronteggiare il disadattamento ma di prevenirlo fin dall'inizio, seguendo insegnanti e allievi soprattutto a livello della comunicazione e dei rapporti interpersonali. Il sostegno pedagogico, oltre ad approfondire la diagnostica dei casi segnalati e a intervenire sugli allievi, cercherà sempre più di interferire con il funzionamento dell'insegnamento ma incontrerà ovviamente grosse resistenze. Offrire collaborazione ai docenti segnalando nel contempo che vi sono rapporti disturbati fra le componenti, non poteva che suscitare perplessità e sfiducia nei confronti di questi nuovi specialisti che pretendevano di sapere meglio degli insegnanti come fare scuola! L'attività di consulenza ai docenti, le offerte di collaborazione per la produzione di soluzioni didattiche adattate alle caratteristiche degli allievi in diffi- 
coltà, le pianificazioni dei lavori nei consigli di classe, ecc. non troveranno l'esito sperato né nelle scuole elementare, né tanto meno nelle scuole medie. Le direzioni degli istituti sono state in grado solo parzialmente di assumere in modo propositivo la finalità di ridurre il disadattamento e ciò a causa di una formazione insufficiente, che le ha portate a ricercare soluzioni immediate a problemi complessi, e del predominio degli aspetti di gestione corrente e amministrativa della scuola che non lascia loro il tempo di riflettere con calma ai problemi pedagogici.

Di fronte alle difficoltà di spiegazione delle strane differenze di comportamento e di rendimento degli allievi, gli operatori scolastici si sono costruiti delle rappresentazioni sociali che le possano chiarire. Le rappresentazioni permettono pure di giustificare le proprie difficoltà di insegnante nell'ottenere gli obiettivi di apprendimento e di salvaguardare una identità sociale gratificante. Mugny e Carugati (1985) affermano che queste rappresentazioni evolvono in funzione delle esperienze vissute e sono diverse a seconda che si tratti di un insegnante in formazione, di un insegnante in attività, con o senza figli propri. L'ideologia del dono, dell'origine naturale della differenza nelle intelligenze risponde all'esigenza di dare una ragione alle difficoltà di apprendimento degli allievi in mancanza di altre spiegazioni più approfondite. Una ricerca svolta da Minoggio (1991) in Ticino sulle rappresentazioni sociali delle cause del disadattamento scolastico mette in luce come la maggioranza non solo dei genitori ma anche degli insegnanti pensino che l'insuccesso sia causato innanzitutto da fattori intellettuali e affettivi interni al soggetto - allievo. Come seconda causa in ordine di rilevanza è citata l'istituzione scolastica per la sua indifferenza alle particolarità individuali e per i ritmi di apprendimento troppo elevati, in terza posizione le ragioni familiari (ambiente di vita poco stimolante, problemi interni alla coppia genitoriale, ecc.). Ritroviamo dunque le principali interpretazioni già rilevate altrove: dalla concezione del dono, per cui le capacità dell'allievo sono il frutto dell'eredità, della genetica, ecc., alla concezione della privazione culturale, dell'origine socio-culturale ed infine la concezione secondo cui la selezione scolastica avviene sulla base di norme o valori socialmente e storicamente definiti (anche se spesso non espliciti) e quindi, almeno in parte, controllabili e modificabili dall'istituzione stessa e dai suoi membri.

\section{L'assestamento dei servizi di sostegno (anni '80 e '90)}

Un tentativo di esplicitare un modello di funzionamento più sistemico dei servizi di sostegno, in particolare nel complesso mondo della Scuola media, avviene con il concetto di inevitabile ventaglio degli interventi del sostegno pedagogico (Dozio 1990) che risultano necessariamente diversificati vista la molteplicità delle funzioni attribuite al sostegno. Si possono comunque distinguere due principali livelli di lavoro: 
1. il livello degli interventi nell'istituzione, di prevenzione o di consulenza alle famiglie, alle direzioni degli istituti e ai docenti, ai quali si aggiunge una prima modalità di intervento più diretto, ma sempre ancora inserito nell'ambito istituzionale della classe o dei consessi interni alla scuola;

2. il livello degli interventi diretti con gli allievi che vanno dal più tradizionale recupero o aiuto scolastico al sostegno all'adattamento scolastico (comprensione delle regole del gioco scolastico, chiarificazione emotiva o delle relazioni, ecc.), all'aiuto più cognitivo-motivazionale all'apprendimento, per finire con le forme di sostegno psicologico alla persona-allievo.

Nel primo decennio del Servizio le segnalazioni degli allievi problematici e gli interventi venivano generalmente condotti secondo il modello medico della segnalazione o della ammissione, del bilancio o della diagnosi con l'uso di strumenti anche psicometrici, seguito da un progetto e dallo svolgimento dell'intervento ed infine la dimissione eventuale del caso. Un cambiamento di paradigma avviene con il riferimento alle teorie sistemiche (Selvini Palazzoli 1981, Evequoz 1984). Si cerca di superare la logica dell'ammissione e della dimissione considerando il sostegno una risorsa per tutti gli allievi, anche se non segnalati esplicitamente, per i genitori e per l'istituzione, e di potenziare le collaborazioni in classe, le diversificazioni curricolari e di indicare ai docenti gli aspetti cognitivi e sociali più promettenti di successo. Si rinuncia all'idea di identificare la causa delle difficoltà per cercare di cogliere le interrelazioni fra le componenti che agiscono sull'allievo portatore del sintomo. Nel caso di un bilancio sulle capacità degli allievi, si cerca di evitare la diagnosi per privilegiare la messa in luce dei potenziali di sviluppo (Bosco 1992, Büchel 1995, Dozio 1987,1988) e di unire al momento diagnostico una fase di apprendimento didattico allo scopo di realizzare al meglio le possibilità di evoluzione del soggetto.

Negli stessi anni viene ribadito il concetto secondo cui il disadattamento è un problema di tutti gli operatori scolastici, docenti e direzioni comprese; non può quindi essere delegato a un Servizio anche se rimane molto diffusa la speranza di un magico e rapido ri-adattamento degli allievi segnalati. L'assunzione della proprie responsabilità da parte della comunità educante, la presa di coscienza e la modifica delle prassi generatrici di disadattamento, rimangono ancora auspici di non facile realizzazione (Collegio dei capigruppo SSP/SM, 1992).

Se negli anni '80 l'insuccesso era visto come qualcosa di riducibile e fors'anche di eliminabile, negli anni '90 la speranza di annullare l'insuccesso è venuta meno poiché ci si è resi meglio conto della doppia funzione della scuola, formativa certamente, ma anche selettiva, normativa e qualificatrice (Perrenoud 1991d, Cardinet 1988). Oltre a far fronte alla richiesta di migliorare i risultati scolastici degli allievi in difficoltà, l'azione degli operatori del sostegno si è concentrata sugli spazi interni all'istituzione nei quali fosse possibile indurre un miglioramento delle condizioni di apprendimento, quali un miglior uso dell'ora di classe, la differenziazione dell'insegnamento, l'intervento svolto direttamente in classe (Ghisla 1987, Cattaneo \& Dozio 1989, Dozio 1995), ecc. Con la na- 
scita dei progetti educativi di istituto (Dozio, 1998), l'istituzione scolastica ha cercato di stimolare un miglioramento delle condizioni di vita e di lavoro interne agli istituti, con l'intento di favorire migliori condizioni di apprendimento e un interesse verso i problemi del disadattamento concepito come uno dei riflessi dei problemi della scuola.

\section{Le trasformazioni dei bisogni sociali degli anni 190}

Agli inizi degli anni '90 la scuola ticinese è in una posizione ambigua che tuttora perdura: da una parte vi è una certa fierezza per gli strumenti messi a disposizione per far fronte al disadattamento e che rimangono espressione di lungimirante coscienza politica e pedagogica, spesso modello per altri cantoni, dall'altra parte la routine dei servizi nella scuola fa fatica a produrre un lavoro effettivamente orientato in modo sistemico e complessivo; mancano inoltre la creazione di strumenti di lavoro e di diversificazione dell'offerta in funzione di una domanda che cambia, la verifica delle misure adottate e la ricerca di una maggiore efficacia.

Il SSP contiene il disadattamento e l'insuccesso, garantisce che qualcuno sia sempre a disposizione per predisporre degli interventi, ma non riesce a coinvolgere il sistema scuola nella differenziazione dell'insegnamento. Nel 1991 Ph. Perrenoud a Lugano affermava che «le principal atout du soutien, aujourd'hui encore, c'est d'être praticable. Alors qu'une vraie différenciation de l'enseignement reste encore de l'utopie» (Perrenoud, 1991d).

Se esaminiamo più da vicino il caso della Scuola media, il SSP è stato creato come risorsa che permettesse a una scuola fondata sul principio dell'integrazione di funzionare e di trovare soluzioni pedagogiche, didattiche e istituzionali conformi alla sua finalità generale. Il pendolo delle scelte pedagogiche, sociali ed ideologiche che si muove fra integrazione e separazione ha continuato ad oscillare. Non tutti gli operatori scolastici si sono convinti delle finalità dell'integrazione e le difficoltà di attuazione del modello si sono manifestate ancor più quando nuove condizioni sociali sono apparse.

La società ticinese si è trovata in questi ultimi anni di fronte a una trasformazione economica e sociale rapida e problematica che ha posto una serie di nuove condizioni all'istituzione scolastica. Il mercato del lavoro richiede personale sempre più formato e le famiglie degli allievi riversano l'aumentata esigenza di livelli di conoscenza e di formazione in una scuola che sta ancora cercando i mezzi per fornire un insegnamento adatto ad allievi diversi fra di loro. Sia i genitori sia gli allievi cercano di evitare i curricoli che danno minori sbocchi verso gli studi superiori, ci si vuole garantire la possibilità di proseguire la formazione per accedere ai posti di lavoro in posizione di vantaggio. La richiesta di una formazione scolastica elevata e di una buona riuscita degli allievi non si limita più ai ceti sociali 
medi e alti, ma si estende all'intera popolazione. L'insuccesso scolastico o un insegnamento ritenuto non soddisfacente crea reazioni immediate. La necessità di ottenere un successo scolastico, formale e sostanziale, con il suo corollario di speranza di riuscita sociale per i figli, si è estesa a tutti gli ambienti sociali e professionali. Vi sono poi i nuovi immigrati, gli alloglotti, i quali occupano la parte bassa della curva della riuscita scolastica e rendono più eterogenee le condizioni di insegnamento. Sono questi ultimi ceti a risentire più di tutti delle difficoltà di integrazione ai valori e alle esigenze del sistema scolastico. Eppure le percentuali delle bocciature nella scuola in generale, e nella scuola media in particolare, restano grosso modo costanti da anni fra il 2 e il $4 \%$.

Se osserviamo i tassi di successo nella Scuola media (DIC, 1999), rilevabili tramite la percentuale di frequenza dei corsi attitudinali (in media il $50 \%$ degli allievi li frequenta) e il diritto d'accesso alle Scuole medie superiori (attorno al $45 \%$ ), emerge un profilo piuttosto positivo del rendimento degli allievi secondo il giudizio dei docenti. Anche le percentuali di promossi senza insufficienze nelle varie classi sembrano piuttosto soddisfacenti oscillando fra il 60 e il $70 \%$. Il diritto di accesso alle Scuole medie-superiori è raggiunto dal 46\% degli allievi ( $51 \%$ delle femmine e $41 \%$ dei maschi) mentre era del $43 \% 10$ anni or sono ( $49 \%$ femmine, $37 \%$ maschi).

\section{Dagli indicatori di insuccesso ad una scuola della riuscita}

Un indicatore che sintetizza l'aspetto istituzionale dell'insuccesso scolastico, è il numero delle note insufficienti accumulate dagli allievi nelle varie materie. Questo indicatore rimane alto nella scuola media per i corsi differenziati a livelli: matematica, francese e tedesco hanno fra il 13 e il $20 \%$ di allievi che registrano valutazioni insufficienti.

Vi è però una percentuale ridotta, ma significativa, di allievi che cumulano un numero consistente di note insufficienti: circa il 12\% degli allievi di Scuola media hanno due o più note insufficienti. La variazione fra gli istituti rimane notevole (Dozio, 2000); i dati del 1998-'99 indicano come:

- la deviazione standard delle note insufficienti attribuite agli allievi che frequentano in IV Scuola media tutti e tre i corsi attitudinali, è del 9,72\% (in valore assoluto da un minimo del $32 \%$ in un paio di istituti a un massimo del 69\%),

- la deviazione standard per la percentuale degli allievi che ottengono il diritto di accesso diretto alle Scuole medio-superiori è del 9,83\% (in valore assoluto da un minimo del $28 \%$ a un massimo del $65 \%$ ),

- la deviazione standard per la percentuale di allievi con più di 2 note insufficienti è del 4,05\% (in valore assoluto da un minimo del 3\% ad un massimo del 19\%).

$\mathrm{Ci}$ si può quindi chiedere se esista una percentuale inevitabile di insuccesso o di disadattamento personale o sociale nella scuola, indipendente dalle variazioni 
delle richieste sociali, dalla popolazione scolastica e dalle riforme di curricolo o pedagogiche introdotte. Esiste una percentuale fisiologicamente necessaria affinché si confermi la distribuzione degli allievi secondo la curva di Gauss (Crahay, 1996) oppure le percentuali di riuscita o di insuccesso sono socialmente determinate, legate alla condivisione di una cultura, dei valori di una scuola o di un istituto? I dati dei risultati scolastici nelle diverse scuole medie mostrano delle tendenze piuttosto costanti nelle sedi, ma con differenze notevoli fra un istituto e un altro. I fattori che spiegano queste variazioni sono certamente multipli, dal tipo di popolazione che frequenta gli istituti, al valore sociale attribuito alla formazione scolastica, alla concezione del ruolo formativo o selettivo attribuito alla scuola dagli operatori scolastici che costituiscono una sorta di cultura di istituto relativa alla valutazione e alla selettività. Rimane il fatto che la cultura di istituto definisce largamente i tassi di insuccesso.

La ricerca (in particolare Perrenoud, 1984) ha ormai mostrato come un tasso di insuccesso sia inevitabile, anzi sia necessario per qualificare la scuola. Una scuola senza insuccesso sarebbe rapidamente squalificata socialmente. Ciò dipende in larga parte dalle rappresentazioni sociali attribuite alla scuola. Se alla scuola elementare si chiede di portare tutti gli allievi alla riuscita negli apprendimenti, alla scuola media -prima tappa della scuola secondaria seppure obbligatoria - si chiede di marcare delle distinzioni nelle capacità degli allievi. In altri sistemi scolastici -dei paesi dell'Europa del nord, per esempio (Crahay, 2000)- le rappresentazioni sulla funzione selettiva della scuola secondaria si sono modificate e a tutta la scuola obbligatoria viene chiesto di essere una scuola della riuscita.

Se l'insuccesso può essere registrato dal confronto dei risultati di un allievo con quelli della maggioranza, il disadattamento può essere definito come una mancata corrispondenza fra la prestazione di un allievo rispetto alla norma stabilita, sia in termini di comportamento relazionale o sociale, sia di risultati scolastici. Nel corso degli anni di esperienza del sostegno, si è fatta strada però un'altra interpretazione per la quale il disadattamento sarebbe meglio definibile facendo riferimento al sentimento, più qualitativo, di malessere o di sofferenza dell'allievo. Questo sentimento può derivare da ragioni molteplici, fra le quali ad esempio la non corrispondenza fra le proprie attese e gli esiti ottenuti, la perdita di fiducia in conseguenza dei risultati scolastici, il vissuto familiare o sociale conflittuale o poco gratificante, ecc. L'insuccesso è in ogni caso in relazione ai risultati scolastici mentre il disadattamento può essere sia dipendente che indipendente dalla situazione scolastica. Vi può essere insuccesso senza sentimento di disadattamento (l'allievo è tranquillo con sé stesso pur avendo cattivi risultati) ma anche disadattamento senza insuccesso (l'allievo che soffre a causa di problemi familiari pur riuscendo a scuola, oppure l'allievo che è più che discreto a scuola ma la cui famiglia esige ancora di più da lui senza che egli senta di poter soddisfare queste ambizioni).

E' possibile sapere qual è l'entità dell'insuccesso e del disadattamento nella scuola media ticinese? La risposta non è ovvia e probabilmente nemmeno possi- 
bile in termini precisi, considerati gli indefinibili confini del fenomeno stesso. Sappiamo però che gli allievi che entrano in contatto diretto con gli operatori del Servizio (segnalati nelle statistiche come seguiti dal SSP) sono circa il 13\% in media sui 4 anni. Ciò significa che il 13\% degli allievi ha delle difficoltà nel corso della scolarità o che il disadattamento è del 13\%? Al Servizio giungono spesso ancora richieste di restaurazione della normalità; le differenze impediscono dunque veramente lo svolgimento dell'attività didattica? Non è possibile superare il senso di insoddisfazione dato da certi allievi che non raggiungono gli obiettivi prefissati, con una diversificazione delle attività proposte in classe, andando oltre l'idea che a tutti gli allievi vada rivolto lo stesso messaggio e che tutti debbano fornire un prodotto analogo? L'intervento di sostegno è spesso, anche se non sempre, visto come un intervento riparatore, un sorta di soccorso della strada scolastica, che ripara il veicolo-allievo e lo rimette nel flusso della circolazione normale. Il riferimento ad un ideale di allievo medio, se non di un buon allievo, mantiene un ruolo centrale nelle concezioni dell'insegnamento, sia dei genitori, sia di una buona parte dei docenti. Nonostante il lavoro di sensibilizzazione del corpo insegnante svolto dagli operatori del Servizio, le differenze fra gli allievi sono ancora viste come un ostacolo al buon funzionamento dell'insegnamento e non come una ricchezza da sfruttare; nonostante il numero importante di ricerche che hanno mostrato le possibilità formative offerte dall'eterogeneità, si continua a preferire l'omogeneità (Crahay 1996). Purtroppo però l'omogeneità è solo un'astrazione. Ammettiamo però per un momento che il servizio offerto agli allievi sia efficace e che riesca a rinormalizzare gli allievi segnalati. Partendo dalla considerazione che la scuola ha bisogno di una parte di insuccesso per qualificarsi, c'è da chiedersi: chi occuperà il posto degli allievi recuperati? Finché la scuola avrà bisogno di un primo e di un ultimo, quale funzione può avere il Servizio di sostegno pedagogico su questo piano?

Non tutti gli operatori scolastici, genitori e allievi si pongono però su posizioni analoghe sul senso delle differenze, delle difficoltà di apprendimento e sui mezzi per ridurre il disadattamento. In effetti, docenti, genitori e allievi indirizzano al Servizio delle domande che oscillano, alla maniera di un pendolo, fra due poli: il polo dell'integrazione e il polo della selezione, della segnalazione della distanza dalla norma. Di fronte alle domande di intervento, l'operatore di sostegno deve scegliere fra priorità opposte:

1. dal punto di vista della scuola: preferire un lavoro sugli apprendimenti previsti dal programma scolastico, oppure promuovere lo sviluppo di competenze più generali, trasversali alle discipline?

2. dal punto di vista dei genitori: mirare al raggiungimento di una certificazione scolastica e sociale soddisfacente, all'ottenimento di note positive, oppure piuttosto allo sviluppo della personalità dell'allievo e delle sue risorse?

3. dal punto di vista degli allievi: mirare piuttosto alla riuscita a corto termine, all'ottenimento di note sufficienti, oppure alla comprensione più profonda, a una formazione che abbia valore a lungo termine? 
L'efficacia delle misure di sostegno, individuali o in classe, non è unanimemente riconosciuta dalle ricerche svolte, in particolare si ricordano R. Slavin e N.Madden (1986), G. Bless (1990), P.-A. Doudin (1996) e D. Martin (1996) e i numerosi contributi di Ph. Perrenoud (1990, 1991a, 1991b,1991c). Per alcuni, ogni esclusione dalla classe ha effetti negativi, per altri il sostegno avrebbe effetti positivi. L'esperienza ticinese mostra come ci siano allievi che approfittano molto di un intervento individualizzato, sia sul piano dell'apprendimento scolastico, sia sul piano più psicologico, motivazionale o sociale. Per difficoltà più scolastiche, un intervento in classe o con piccoli gruppi può essere utile, ma è pure indispensabile un dialogo e una negoziazione con il docente, vale a dire un chiarimento del problema incontrato dall'allievo, la progettazione di attività didattiche favorevoli per un apprendimento efficace, il chiarimento delle esigenze che saranno oggetto di valutazione, l'esplicitazione delle competenze di cui comunque l'allievo dispone anche se lo si ritiene in situazione di insuccesso o di disadattamento. Una collaborazione in classe è da prevedere sia per l'osservazione delle situazioni problematiche, sia per un lavoro in comune con gli insegnanti di materia. E' in genere però ancora con misure esterne all'insegnamento, di recupero scolastico, che si ricerca la soluzione riaffermando il principio che la causa delle difficoltà risiede nell'allievo o nell'ambito familiare. Ambiti di partenariato fra scuola e famiglia, un lavoro comune fra i docenti che insegnano nella stessa classe, funzioni di tutorato agli allievi sono ancora piste poco esplorate.

\section{L'evoluzione delle richieste di formazione}

In questi ultimi anni, assistiamo ad alcuni cambiamenti sociali che hanno e avranno pesanti conseguenze sull'accettabilità per la popolazione del Cantone dell'idea di insuccesso e di disadattamento scolastico. Alcuni fenomeni sociali indicano un aumento dell'importanza della formazione scolastica e una richiesta più complessa nei confronti di quest'ultima. In particolare:

1. la generalizzazione dell'importanza della riuscita scolastica spesso ora anticipata alla scuola elementare o alla scuola dell'infanzia e preparata e stimolata dai genitori nelle attività extrascolastiche previste per i figli. La richiesta, per una discreta percentuale di allievi, di posticipare l'inizio della scolarità per garantire un più sicuro successo, potrebbe esserne un indicatore;

2. il rafforzamento dell'idea di un'educazione d'élite, d'altronde mai del tutto sopita. Se in genere vi è accordo sulla necessità di compiere degli sforzi sociali e didattici per aiutare gli allievi meno favoriti, non si vuole dimenticare la maggioranza degli allievi che deve beneficiare della scuola per prepararsi ad entrare in un mondo altamente competitivo (ad es. Guntern, 1998) e neppure il problema della differenziazione dell'insegnamento per gli iperdotati (Besse, 2000); 
3. la richiesta sociale e del mondo dell'economia di non insistere più sull'acquisizione di conoscenze ma piuttosto di atteggiamenti e di abilità di valore generale e trasversale alle tradizionali materie di insegnamento;

4. il multiculturalismo e multilinguismo sempre più marcato $(29 \%$ di allievi stranieri nelle scuole obbligatorie nel '97-98, il $20 \%$ di lingua materna non italiana e di questi quasi il $2 \%$ allievi alloglotti da non più di 2 anni in Ticino);

5. attribuzione alla scuola di compiti formativi precedentemente assunti da altre agenzie educative oggi più latitanti. La disgregazione della famiglia nucleare $\mathrm{e}$ la crescente incertezza nel quadro dei valori e delle norme tradizionali, si riflettono sulle condizioni del lavoro scolastico. Si denunciano ormai spesso atti di indisciplina, di inciviltà o di violenza all'interno degli Istituti da parte di preadolescenti.

Questa diversificazione delle funzioni, per certi versi divergenti fra loro, pone la prevenzione dell'insuccesso e del disadattamento in una nuova ottica. Le tendenze che si stanno affermando, sostengono che non solo si tratta di sostenere l'allievo nella sua frequenza scolastica, ma di sostenere l'istituzione nella diversificazione delle sue funzioni. La scuola avrebbe oggi il compito di assumere di nuovo una funzione formativa più globale e profonda che rimane in secondo piano quando ancora prevale la trasmissione disciplinare. Per evitare la demotivazione di gruppi di allievi o il loro insuccesso, sarebbe necessario proporre un insegnamento differenziato attento all'apprendimento anche degli allievi deboli e di altre etnie. Nel contempo dovrebbe stimolare gli allievi brillanti, creare condizioni per un dialogo fra docente e allievi che vada oltre l'insegnamento in senso stretto, suscitare il piacere di stare a scuola, cercare di far acquisire competenze valide al di là dell'ambito scolastico. Sono questi però compiti che i docenti non si sentono di affrontare da soli. Il lavoro educativo e formativo esige un progetto comune, di istituto, che coinvolga le diverse componenti della scuola ma anche il Servizio di sostegno. E' l'istituzione scuola nel suo insieme che dovrebbe, nelle sue complesse interazioni, riuscire a generare le condizioni per una riuscita scolastica degli allievi. Gli operatori di sostegno potrebbero essere allora chiamati a collaborare nell'impostazione del clima interno agli istituti e nelle classi, in un lavoro comune con i docenti al fine di differenziare l'insegnamento e di creare condizioni di benessere.

\section{Conclusioni}

Nel corso degli ultimi cinquant'anni, il Ticino è passato a una economia terziarizzata, a un sistema scolastico integrato, a una assunzione esplicita del disadattamento e si è confrontato con problemi non dissimili da quelli presenti nel resto dell'Europa. L'intervento sull'insuccesso e il disadattamento, dapprima collegato all'insegnamento speciale, alla psicopatologia e alla pedagogia curativa, è stato in 
seguito influenzato dalla lettura sociologica in termini di riproduzione sociale e di distanza socio-culturale. Le cause una volta attribuite alla biologia dell'allievo hanno poi integrato la famiglia, l'ambiente sociale ed infine anche la scuola stessa come rivelatrice e generatrice di disadattamento. A partire da una visione normativa della scuola si è sviluppata, ma non si è ancora affermata, una visione che privilegia la positività delle differenze, la ricchezza dell'interculturalità e del multilinguismo, la possibilità di lasciare all'allievo un tempo per l'apprendimento valutato in modo formativo. Da un sistema scolastico centralizzato sono emerse una tendenza alla valorizzazione delle realtà locali, delle dinamiche dei singoli istituti. All'interno degli istituti stessi vengono messi lucidamente in rilievo quelle componenti e quei comportamenti che sono generatori di problemi, ma spesso non si sa come intervenire per porvi rimedio. Alcune componenti del sistema scolastico hanno compreso l'importanza della posta in gioco, ma la trasformazione di un sistema così storicamente definito non può che avvenire per mezzo di innovazioni che impongono dei cambiamenti che sono di fatto non solo tecnici ma soprattutto culturali. Nel contempo non è sufficiente pensare che gli operatori del sostegno pedagogico possano assumere la responsabilità della riduzione dell'insuccesso e del disadattamento senza una modifica complessiva delle concezioni dell'insuccesso, delle pratiche didattiche, delle relazioni con le famiglie e con gli altri servizi esterni alla scuola. Una scelta politica compiuta ormai alcuni decenni fa e confermata per il seguito, è oggi da rileggere alla luce del cambiamento del contesto sociale ed economico e dei bisogni derivanti, così come dei paradigmi esplicativi delle scienze dell'educazione. Il Canton Ticino dovrebbe quindi darsi il compito di ri-valutare il lavoro svolto nella prevenzione del disadattamento, di definire più precisamente gli obiettivi della lotta all'insuccesso, di prevedere quegli aggiustamenti nei mezzi a disposizione del Servizio e degli Istituti che permettano agli operatori scolastici di disporre di capacità e risorse adeguate per agire in maniera coordinata nella complessa realtà nella quale sono chiamati ad operare. In caso contrario, il rischio è di lasciare a metà un lavoro che era iniziato in maniera promettente.

\section{Riferimenti bibliografici}

Basaglia F. (1973). Cosè la psichiatria. Torino: Einaudi.

Bennati-Besozzi, E. (a cura di) (1979). Rapporto sull'istituzionalizzazione del Servizio di sostegno pedagogico con particolare riferimento alle scuole elementari. Bellinzona: USR/UES/CISE.

Besse Caiazza, Anne-Marie (a cura di di) (2000). La pédagogie spécialisée face aux élèves surdoués. Lausanne: SPC.

Bless, G. (1990). Intégration et/ou séparation - La scolarisation des élèves ayant des difficultés scolaires. Educateur, 9, 6-10.

Bosco, D. (1992). L'évaluation dynamique du potentiel d'apprentissage. Psychoscope, 9, 4-7.

Büchel, F.-P. (1995). L'éducation cognitive. Le développement de la capacité d'apprentissage et son évaluation. Neuchâtel et Paris: Delachaux et Niestlé. 
Cardinet, J. (1988). Evaluer sans juger. Neuchâtel: IRDP.

Cattaneo, M. \& Dozio E. (1989). L'intervento nella sede come prevenzione. Rivista del Servizio di sostegno pedagogico, 4, 45-52.

Collegio dei capgruppo SSP/SM, (1992). Il disadattamento è un problema di tutti. Rivista del Servizio di sostegno pedagogico, 9, 58-61.

Commissione cantonale di coordinamento dei Servizi di sostegno pedagogico (1987). Dati statistici relativi agli allievi seguiti dai servizi di sostegno pedagogico - anno scolastico 1985/86. Bellinzona: Ufficio studi ricerche.

Commissione speciale scolastica (1984). Rapporto sul messaggio del 13 giugno 1984 concernente l'istituzione del sostegno pedagogico. Bellinzona: Segreteria del Consiglio di Stato.

Consiglio di Stato del Canton Ticino (1957). Messaggio del Consiglio di Stato al Gran Consiglio accompagnante il progetto di una nuova legge scolastica. Bellinzona: CdS.

Consiglio di Stato del Canton Ticino (1972). Listituzione della scuola media nel Canton Ticino, messaggio del Consiglio di Stato al Gran Consiglio e disegno di legge. Bellinzona: Dipartimento della pubblica educazione.

Consiglio di Stato del Canton Ticino (1984). Messaggio concernente l'istituzione del sostegno pedagogico del 13 giugno 1984. Bellinzona: CdS.

Crahay, M. (1996). Peut-on lutter contre l'échec scolaire? Bruxelles: De Boeck.

Crahay, M. (2000). L'école peut-elle être juste et efficace? De l'égalité des chances à l'égalité des acquis. Bruxelles: De Boeck.

DPE - Dipartimento Pubblica Educazione (1971). Censimento della popolazione scolastica del Canton Ticino. Bellinzona: Ufficio studi e ricerche.

DIC - Dipartimento Istruzione e cultura (1999). Statistiche sulla scuola media. Bellinzona: Ufficio Insegnamento Medio.

DIC - Dipartimento Istruzione e cultura (2000). Statistica degli allievi fine anno 1998-99 Bellinzona: Ufficio studi e ricerche.

Doudin, P.A. (1996). Elèves en difficulté. La pédagogie compensatoire est-elle efficace ? Psychoscope, 9, 4-7.

Dozio, E. (1987). Sei immagini in cerca di uno scopo, Rivista del Servizio di sostegno pedagogico della Scuola media, 2, 6-13.

Dozio, E. (1988). La mappa mentale nel primo incontro, Rivista del Servizio di sostegno pedagogico, 3, 21- 25.

Dozio, E. (1990). Linevitabile ventaglio. Rivista del Servizio di sostegno pedagogico, [Monografia].

Dozio, E. (1993). L'immagine di sé negli allievi scolasticamente più deboli. Rivista del Servizio di sostegno pedagogico, 10, 5-36.

Dozio, E. (1995). Intervento in classe e dintorni. Rivista del Servizio di sostegno pedagogico, 13, 28-33.

Dozio, E. (1998). Verso il progetto educativo di istituto. Rivista del Servizio di sostegno pedagogico, 16, 13-42.

Dozio, E. (2000). Il Servizio di sostegno pedagogico della scuola media ticinese, 20 anni dopo. Rivista del Servizio di sostegno pedagogico, 18, 35-50.

Evequoz, G. (1984). Le contexte scolaire et ses ôtages, vers une approche systémique des difficultés scolaires. Paris, ESF.

Ferrari, C. \& Sargenti, W. (1968). Situazione della scolarità speciale nel Cantone e proposte per un programma di realizzazione. Bellinzona: Dipartimento della Pubblica Educazione.

Ghisla, G. (1987). L'intervento in classe: una prospettiva interessante. Rivista del Servizio di sostegno pedagogico, 2, 6-7.

Gruppo sperimentale del VI circondario di scuola elementare (1982). Rapporto conclusivo relativo alla verifica della sperimentazione del Servizio di sostegno. Locarno: Ispettorato scolastico.

Guntern, G. (1998). Nous avons besoin des élites. Educateur, 6, 4-7.

Martin, D. (1996). Lutter contre l'échec scolaire. Psychoscope, 9, 8-10.Martinoni, M. (1990). Les difficultés d'apprentissage. In A. Bürli A. \& G. Sturny-Bossart (Ed.), Annuaire 1988/89 sur la pédagogie spécialisée en Suisse (pp. 25-33). Lucerne: Edition SZH/SPC. 
Merzaghi, G. (1997). L'allievo in difficoltà, tra modelli integrativi e modelli esclusivi. Luzern: Edition SZH/SPC.

Minoggio, W. (1991). Le rappresentazioni sociali del disadattamento scolastico. Bellinzona: Ufficio studi e ricerche del DIC.

Mugny, G. \& Carugati, F. (1985). L’intelligence au pluriel. Les représentations sociales de l'intelligence et de son développement. Cousset: Delval.

Perrenoud, Ph. (1984). La fabrication de l'excellence scolaire: du curriculum aux pratiques d'évaluation. Vers une analyse de la réussite, de l'échec et des inégalités comme réalités construites par le système scolaire. Genève: Droz.

Perrenoud, Ph. (1990). Come combattere l'insuccesso scolastico in dieci lezioni.... Scuola Ticinese, 163, 3-8, (traduzione della comunicazione al congresso «L'avenir du système éducatif français, axes de recherche», Paris, 1985).

Perrenoud, Ph. (1991a). Du soutien pédagogique à une vraie différentiation de l'enseignement: évolution ou rupture? Genève: FPSE, Service de la recherche sociologique.

Perrenoud, Ph. (1991b). L'échec scolaire vous dérange? Il y a peut-être quelque chose à faire. Conférence Ottawa.

Perrenoud, Ph. (1991c). Le soutien pédagogique, une réponse à l'échec scolaire? Genève: FPSE, Service de la recherche sociologique.

Perrenoud, Ph. (1991d). Du soutien pédagogique à une vraie différenciation de l'enseignement: évolution ou rupture. Genève: Service de la recherche sociologique \& Faculté de psychologie et des sciences de l'éducation.

Pierrehumbert, B. (sous la direction de) (1992). L'échec à l'école: échec de l'école? Neuchâtel \& Paris: Delachaux et Niestlé.

Selvini Palazzoli, M. (a cura di) (1981). Sul fronte dell'organizzazione, strategie e tattiche. Milano: Feltrinelli.

Slavin, R.E. \& Madden N.A. (1986). L'intégration des élèves en difficulté dans les classes ordinaires. Perspectives, XIV (4), $489-509$.

Sturny-Bossart, G. (1996). Toujours plus d'élèves dans les classes de développement et les écoles spéciales. Pédagogie spécialisée, 1, 9-13.

Sturny-Bossart, G. (2000). Le soutien pédagogique dans les cantons suisses: options politiques, choix pédagogiques et problèmes actuels. Rivista del Servizio di sostegno pedagogico, 18, 11-25.

Thurler, M. (1980). Rapporto intermedio sulla sperimentazione del sostegno pedagogico nelle scuole medie. Bellinzona: Dipartimento della Pubblica Educazione.

\section{Politische Massnahmen im Bereich des Schulversagens und der gescheiterten Integration: Entwicklungen im Tessin}

\section{Zusammenfassung}

Der Artikel untersucht die Entwicklung der Schulpolitik im Kanton Tessin in den letzten 50 Jahren im Bereich des Schulversagens und der Schwierigkeiten im Lern- aber auch im sozialen und affektiven Verhalten innerhalb der Institution. Diese Zeitspanne ist vor allem durch eine bedeutsame demographische Entwikklung und tiefgreifende wirtschaftliche Veränderungen gekennzeichnet. Das gewählte Schulsystem zielt auf Integration, was die Organisation vor allem der Sekundarstufe I stark geprägt hat. Die Demokratisierung des Bildungssystems hat dabei die Beziehung der Tessiner Gesellschaft zur Schule verändert; die Reform 
der Sonderschule und der Aufbau des Servizio di sostegno pedagogico (Pädagogischer Betreuungsdienst) brachten ihrerseits eine neue Sichtweise auf die Lernunterschiede und das Schulversagen mit sich. In den letzten 30 Jahren haben diese Servizi sich durchgesetzt und die Auffassungen und die Praxis in Fragen der Integration haben sich stetig verändert; das Bedürfnis nach weiteren Entwicklungen bleibt jedoch bestehen.

\title{
Évolution de la politique scolaire sur l'échec et I'inadaptation: le cas du Canton du Tessin
}

\begin{abstract}
Résumé
L'article décrit l'évolution de la politique sur l'échec et l'inadaptation dans les écoles du Canton du Tessin pendant les dernières 50 années. Dans ce laps de temps, il y a eu une importante évolution démographique et une profonde transformation économique. Le système scolaire adopté a été de type intégratif et cela a profondément influencé surtout l'organisation du secondaire inférieur. La démocratisation de l'accès aux études a d'abord modifié le rapport entre la société tessinoise et l'école, la révision de l'enseignement spécialisé et la création du Service de soutien pédagogique ont ensuite introduit une nouvelle vision des différences dans l'apprentissage et de l'échec scolaire. Dans les dernières 30 années, les Services créés se sont stabilisés et les conceptions et les pratiques autour de l'inadaptation scolaire ont continuellement évolué; néanmoins de nouvelles exigences de changement s'annoncent.
\end{abstract}

\section{The Evolution of School Policy on failure and underachievement: the case of Canton Ticino}

\footnotetext{
Abstract

The article traces the evolution of educational policy on school failure and underachievement due to different causes in Canton Ticino during the last 50 years: period when the area underwent important demographic and economic change. The educational system chosen was headed by the concept of integration and this has had radical effects on the organization of the lower secondary school. The democratization of education has changed the relationship between society and school and the reform of special educational needs and the setting up of a service of pedagogical help ( Servizio di sostegno pedagogico ) led to a new perception of what differences in learning ability and failure in school could mean. During the last 30 years the available special services have been reinforced and the concepts and practice dealing with underachievement have undergone constant revision. However, the need for further change can be perceived.
} 